\title{
Optical Grooming in OFDM-based Elastic Optical Networks
}

\author{
Guoying Zhang ${ }^{12}$, Marc De Leenheer ${ }^{13}$, and Biswanath Mukherjee ${ }^{1}$ \\ ${ }^{1}$ University of California - Davis, 95616, CA, USA; ${ }^{2}$ China Academy of Telecom Research, 100083, Beijing, China; ${ }^{3}$ Ghent \\ University - IBBT, Belgium; Email: \{zguoying, mleenheer, bmukherjee\} @ucdavis.edu; zhangguoying@catr.cn
}

Abstract: We propose a novel optical grooming approach to aggregate and distribute traffic directly at the optical level in OFDM-based elastic optical network. Significant transmitter and spectrum saving is achieved compared to non-grooming scenario.

OCIS codes: (060.4251) Networks, assignment and routing algorithms; (060.1155) All-optical networks

\section{Introduction}

Recently, a novel OFDM-based elastic optical network architecture [1] was proposed to meet the large capacity and diverse traffic granularity requirements for future Internet [2]. OFDM is a multi-carrier transmission technology that transmits a high-speed data stream by splitting it into multiple parallel low-speed subcarriers [3]. By breaking the fixed grid wavelength allocation limitation of WDM and employing subcarrier multiplexing of optical OFDM technology, a bandwidth elastic optical path can be setup in the elastic optical network using just enough spectrum (subcarriers) according to the transmitted data rate. OFDM-based elastic optical network supports various data rates (including sub-wavelength and super-wavelength services) through data rate and bandwidth-variable (BV) transponders at the network edge and bandwidth-variable optical cross-connects (BV-OXCs) in the network core, as shown in Figure 1.

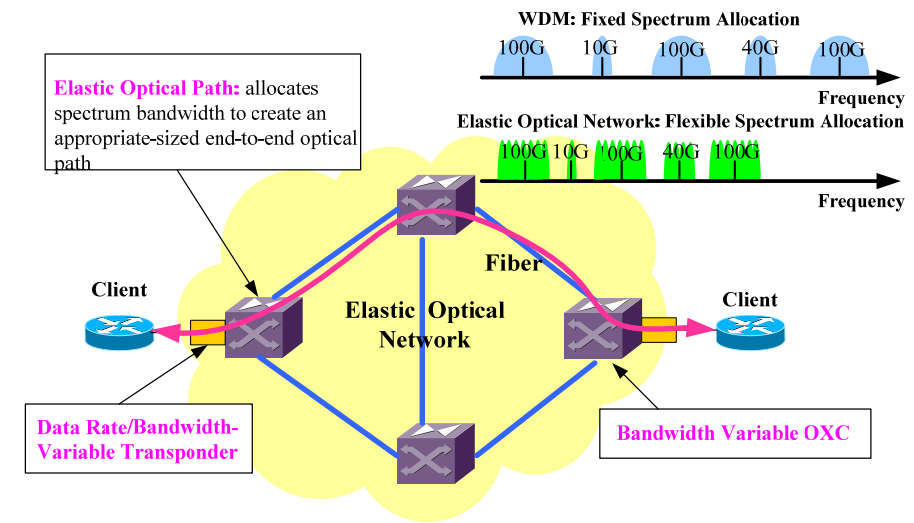

Figure 1. Architecture of elastic optical network.

\begin{tabular}{|c|l|l|}
\hline & \multicolumn{1}{|c|}{ WDM network } & \multicolumn{1}{|c|}{ Elastic Optical Network } \\
\hline $\begin{array}{c}\text { Sub- } \\
\text { wavelength } \\
\text { service }\end{array}$ & $\begin{array}{l}\text { Fixed spectrum } \\
\text { allocated even if not } \\
\text { enough traffic in a } \\
\text { channel, traffic } \\
\text { grooming needed. }\end{array}$ & $\begin{array}{l}\text { Elastic spectrum allocated } \\
\text { according to requested data } \\
\text { rate, sub-wavelength } \\
\text { service supported directly in } \\
\text { optical domain. }\end{array}$ \\
\hline $\begin{array}{c}\text { Super- } \\
\text { wavelength } \\
\text { service }\end{array}$ & $\begin{array}{l}\text { Inverse } \\
\text { multiplexing, } \\
\text { multiple } \\
\text { independent WDM } \\
\text { channels allocated. }\end{array}$ & $\begin{array}{l}\text { Merge several OFDM } \\
\text { channels into a super- } \\
\text { channel, maintain spectrum } \\
\text { orthogonality among the } \\
\text { channels to save spectrum. }\end{array}$ \\
\hline Transponder & $\begin{array}{l}\text { One type of } \\
\text { transponder for one } \\
\text { data rate. }\end{array}$ & $\begin{array}{l}\text { A single type of bit rate and } \\
\text { bandwidth-variable } \\
\text { transponder used for all } \\
\text { service types. }\end{array}$ \\
\hline
\end{tabular}

Table 1. Comparison of WDM and elastic network.

Table 1 shows the differences in service provisioning between WDM network and elastic optical network. In OFDM-based elastic optical network, a single type of bit rate and bandwidth-variable transponder (e.g., 40 Gb/s 400 $\mathrm{Gb} / \mathrm{s}$ ) can be used for all service types. A sub-wavelength service connection is supported directly by a BV transponder and transmitted as an elastic optical path. This scheme is spectrum efficient. However, it might not be cost efficient, since the high capacity of the transponder may not be fully used when carrying sub-wavelength traffic. On the other hand, to switch each sub-wavelength optical path, guard bands are needed between adjacent optical paths because of the imperfect shape of the WSS filter. This may result in a significant amount of spectrum overhead [4], especially when there are a large number of sub-wavelength services.

To address these problems, it is desirable to groom multiple sub-wavelength services into a single BV transponder, and switch them together as a whole. Similar to traditional traffic grooming approach in WDM networks, one possible way to implement traffic grooming in elastic optical networks is to employ electrical subcarrier multiplexing and switching [5]. However, electrical traffic grooming approach adds additional OEO conversions and electrical subcarrier switching requirements to intermediate OXC nodes, which may result in higher cost and energy consumption [6]. These issues may become even more significant when higher speed transmission systems are deployed.

In this paper, we propose a novel optical grooming approach to aggregate and distribute traffic directly at the optical level, and as such, eliminating OEO conversions on intermediate node, by separately switching different OFDM subcarriers originating from the same BV transponder.

\section{Optical Grooming Approach}

The basic idea of optical grooming is to group multiple sub-wavelength optical paths into one BV transmitter. We refer to this group of optical paths as an optical tunnel. Based on the orthogonal feature of OFDM signals, traffic originating from the same source (BV transmitter) can be groomed together without any guard band in between. Using the elastic spectrum switching nature of the BV OXC, a subset of subcarriers in the optical tunnel (corresponding to a 
sub-wavelength optical path) can be dropped or switched optically at any intermediate node along the route. For traffic originating from different source nodes (which use different oscillators), as orthogonality can not be guaranteed between them, they must be received separately using different receivers. Therefore, we only consider source grooming in optical grooming approach.

Normally, guard bands are needed between optical paths, because the imperfect shape of WSS filters may bring in interference from adjacent paths. When employing optical grooming, it is desirable that no guard bands are needed between groomed services, as this leads to inefficient use of transponder and spectrum. Services with the same source and same destination can be switched as a single optical path, therefore no guard bands are needed between them. For services with the same source and different destinations, it is also possible to groom them together without guard bands. As shown in Figure 2, when a sub-wavelength optical path needs to be separated from the optical tunnel at an intermediate node, the original optical tunnel is split into multiple optical tunnels. Guard bands should be added besides the separated optical tunnels, so that they can be switched on subsequent BV-OXCs. In order to switch a subset of the continuous subcarriers in an optical tunnel with the added guard bands to it, it is required that the BV-OXC employs a broadcast-and-select structure [1] (as shown in Figure 3), i.e., the signal can be replicated over different outputs of the OXCs, as in most of the current ROADM implementations. A non-broadcast-and-select OXC structure [7] could also be used, while guard bands need to be put between the different optical paths within a transmitter. To further maximize traffic grooming, we can also groom same source traffic that do not share a common route, meaning that the optical tunnel will split at the orginating OXC.

To realize the optical grooming approach, BV transponders need to be designed to support multi-services, multi-reach, and sometimes guard band in between, which is also refered to as sliceable transponder [8]. These features have already appeared in some recent research works $[9,10]$.

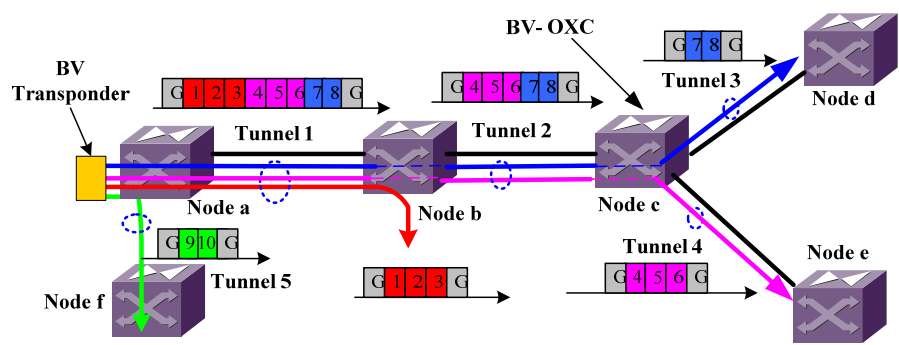

Figure 2. Optical grooming in elastic optical network.

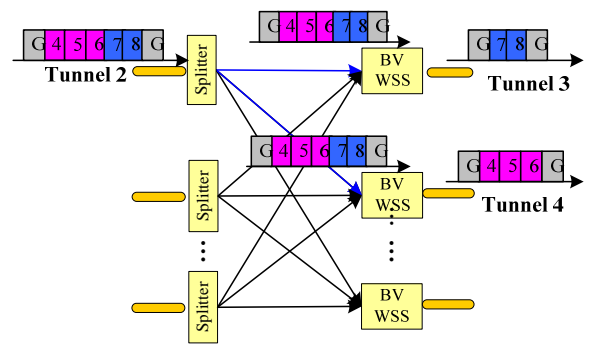

Figure 3. Broadcast-and-select OXC structure.

\section{Optical Grooming Algorithm}

By employing optical grooming, the total number of transmitters can be reduced, and spectrum can be saved because of the eliminated guard bands between groomed sub-wavelength services. However, grooming services into one transmitter adds more constraints to routing and spectrum assignment (RSA) algorithm. We initially formulate the RSA problem of optical grooming using integer linear programing (ILP). We are given a physical topology $G_{p}=\left(V, E_{p}\right)$, where $V$ is the set of network nodes, and $E_{p}$ is the set of unidirectional links. Spectra resources on each link are classified into $W$ spectrum slots, where each slot corresponds to an OFDM subcarrier. We assume BV transponders have a maximum capacity of $T$ subcarriers (coresponds to maximum capacity of an optical tunnel), and guard band between optical tunnels is $2 G B$ (in the number of slots). We are given a traffic matrix, where $\Lambda_{s d}$ is the required capacity between each s-d pair represented by the number subcarriers. Physical layer impairments are not considered in the model for simplicity. The objective of routing and spectrum allocation is to either minimize the number of transmitters or minimize the total spectrum usage. The detailed ILP fomulation is omitted because of space limitations.

As ILP does not scale for large networks, we also proposed two heuristics. The Least Spectrum Grooming (LSG) algorithm minimizes the total spectrum usage by grooming the optical paths that share the longest common route from the source node. The algorithm aims to maximize spectrum gain by the reduced guard bands, and is detailed below. In contrast, the Minimum Transmitter Grooming (MTG) algorithm aims to minimize the number of transmitters. It is largely similar to LSG in terms of algorithmic steps. The difference is that when searching for possible placement of a tunnel, MTG uses maximum spectrum capacity of a tunnel $(T)$ instead of the real capacity of a path (i.e., reserves the spectrum for grooming), and allows to groom paths whose weight (spectrum gain) $H_{s d^{\prime} j}<=0$. The complexity of both algorithms is $\mathrm{O}\left(k N^{3}(\mathrm{ND}+D W+k N)\right)$ for the worst case.

\section{Results and Discussion}

We evaluated the performance of the optical grooming approach and compared to the elastic optical network RSA without grooming [11]. A 5-node mesh network (14 links) and NSF network topology (14 nodes, 44 links) are used for simulation. We assume a spectrum slot width of $12.5 \mathrm{GHz}$, and by adopting QPSK modulation, each subcarrier has a capacity of $25 \mathrm{~Gb} / \mathrm{s}$. Assuming the maximum capacity of a transmitter is $400 \mathrm{~Gb} / \mathrm{s}$ (16 subcarriers), and the guard band size is 2 spectrum slots. Traffic demand for each s-d pair in terms of subcarrier number was randomly generated between 
1 to $D(D=4 \sim 16)$. The number of precalculated paths is 2 .

\section{Least Spectrum Grooming (LSG) Algorithm}

1. Compute k-shortest paths for all s-d pairs, let $P_{s d i}(i \leq k)$ be the ith path in $P_{s d}$.

2. Calculate weight for all s-d pairs, $\mathrm{W}_{\mathrm{sd}}=$ length of $P_{s d i}$.

3. Sort all s-d pairs according to $W_{s d}$ in descending order.

4. Select an s-d pair from the top of the s-d pair list, and set up an optical tunnel $T_{s d}$ as follows:

a) For each shortest path of s-d, search in the network for the first possible placement of $P_{s d i}$ with the capacity of $\left(\Lambda_{s d}+2 G B\right)$.

b) Select the path which has the lowest starting spectrum slot index. If there are multiple candidate paths having the same lowest starting spectrum slot index, select the shortest path.

c) Record the first spectrum slot index $f_{s d}$ and last spectrum slot index $l_{s d}$ of tunnel $T_{s d}$. Record the capacity of the tunnel to be $C_{s d}=\Lambda_{s d}$. Remove all $P_{s d i}$ from the s-d pair list.

5. Construct a possible grooming group $G_{s d}$ for the tunnel

$T_{s d}$, by searching in all s-d paths $\left(P_{s d^{\prime} j}\right)$ that have the same source node $s$. Compare the length of the common continuous route from source node of $T_{s d}$ and $P_{s d^{\prime} j}$, calculate weight $H_{s d^{\prime} j}$ for each $P_{s d^{\prime} j}, H_{s d^{\prime} j}=$ $2 G B \times$ common route length - (length of $P_{s d i j}$ length of $\left.P_{s d^{\prime} 1}\right) \times \Lambda_{s d^{\prime}}$, which represents the spectrum gain by grooming this path. If $H_{s d \prime j}>0$, put $P_{s d \prime j}$ into $G_{s d}$.

6. Sort $P_{s d^{\prime} j}$ in $\mathrm{G}_{\mathrm{sd}}$ by $H_{s d^{\prime} j}$ in descending order.

7. While $C_{s d}<T$ and $G_{s d} \neq \phi$

a) From the top of $G_{s d}$, find a $P_{s d^{\prime} j}$, which can be placed in the network starting from slot index $l_{s d}-G B$, or ending on slot index $f_{s d}+G B$, with capacity $\Lambda_{s d}+2 G B$.

b) If such a demand can be found, then record the new $f_{s d}$ and $l_{s d}$ of the tunnel, and remove s-d' from $G_{s d}$ and s-d pair list.

8. If there are s-d pairs not assigned a route and spectrum yet, then go to 4).

We compared ILP and heuristics on the 5-node mesh network. The differences between the results of ILP and heuristics are within 5 10\%. Figure 4 presents the simulation results of NSF network using the proposed heuristics. We observe that optical grooming achieves significant transmitter savings of around $25 \sim 80 \%$ for different traffic granularities (Fig.4a). Transmitter savings decrease as the traffic granularity grows, as a result of less grooming opportunities for traffic of large granularity. Note that optical grooming saves only transmitters, not the receivers, such that the saving of total number of transmitters and receivers would be half of the percentage shown in the results. Another interesting observation is that optical grooming achieves $4 \sim 15 \%$ spectrum saving compared to the non-grooming scenario by eliminating guard bands within the transmitter (Fig.4b). However, the maximun used spectrum index on each link increased, especially for MSG algorithm (Fig.4c). This demonstrates that arranging optical paths together in a transmitter adds more constraints to spectrum allocation and may lead to more spectrum fragmentation [12] in the network. Therefore, network design should carefully consider the trade-off between minimizing transmitter usage and minimizing spectrum usage.

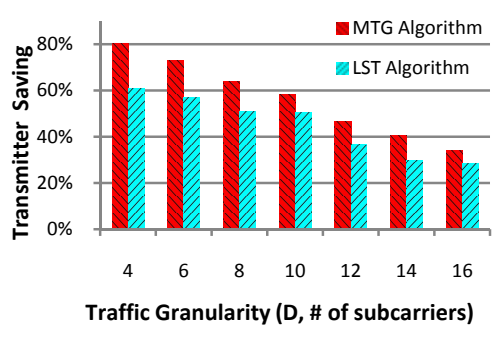

Figure 4 a) Transmitter saving.

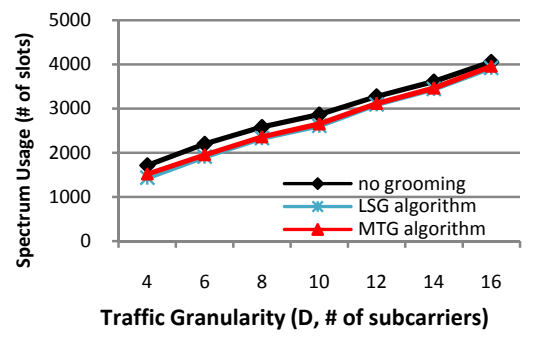

Figure 4 b) Total spectrum usage.

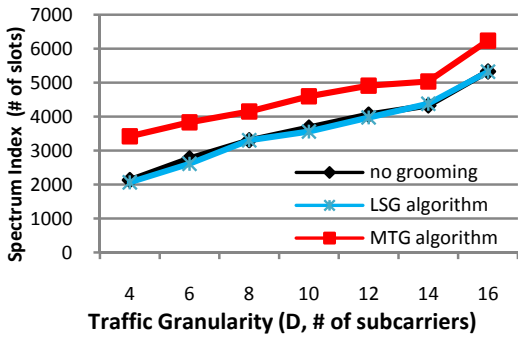

Figure 4 c) Maximum spectrum index.

\section{Conclusion}

From the discussion above, we believe that optical grooming has great potential in cost saving for OFDM-based elastic optical networks. However, more research work on the physical equipment and network design strategies is needed.

\section{References}

[1] M. Jinno et al., IEEE Comm. Mag., vol. 47, no. 11, pp. 66-73, 2009.

[2] E. L. Rouzic et al., Proc., ECOC 2011, Paper Tu.6.K.1.

[3] J. Armstrong, J. Lightwave Technol., vol. 27, no. 3, pp. 525-526, 2009.

[4] A. Morea et al., Proc., OFC/NFOEC 2011, Paper JWA62.

[5] Y. Zhang et al., Proc. OFC/NFOEC 2011, Paper OTuI1.

[6] K. Sato, Proc., ECOC 2011, paper Mo.2.K.1

[7] N. Amaya et al., Proc., OFC/NFOEC 2011, Paper
OMW3.

[8] O. Gerstel, Proc., IPR/PS 2010, Paper PMD3, 2010.

[9] D. J. Geisler et al., Proc., OFC/NFOEC 2011, Paper OTuE3.

[10] H. Takara et al., Proc., ECOC 2011, Paper Tu.5.A.4.

[11] K. Christodoulopoulos et al., Proc., GlobeCom 2010, pp.1-6.

[12] A. N. Patel et al., Proc., OFC/NFOEC 2011, Paper OTuI8. 